\title{
Antiferromagnetic layer thickness dependence of the IrMn/Co exchange-bias system
}

\author{
M. Ali,* C. H. Marrows, M. Al-Jawad, and B. J. Hickey \\ Department of Physics and Astronomy, E. C. Stoner Laboratory, University of Leeds, Leeds LS2 9JT, United Kingdom
}

A. Misra, ${ }^{\dagger}$ U. Nowak, and K. D. Usadel

Theoretische Tieftemperaturphysik, Gerhard-Mercator-Universität Duisburg, 47048 Duisburg, Germany

(Received 22 January 2003; revised manuscript received 22 May 2003; published 16 December 2003)

\begin{abstract}
A study of exchange bias in IrMn/Co systems is presented. Temperature and thickness dependence studies have revealed nonmonotonic behavior in both exchange bias fiel and coercivity with both variables. In particular the exchange bias fiel shows a peak for low IrMn thicknesses that is suppressed at temperatures higher than about $200 \mathrm{~K}$. Calculations using the domain state model of exchange biasing are able to describe all the features seen in the experimental data.
\end{abstract}

PACS number(s): 75.70.Cn, 75.60.-d, 75.50.Lk

\section{INTRODUCTION}

Although the discovery of exchange bias by Meiklejohn and Bean ${ }^{1}$ was over 40 years ago, the effect is still being investigated by the scientifi community to understand the fundamental mechanisms controlling both the exchange bias fiel $H_{\mathrm{ex}}$, and the coercivity $H_{\mathrm{c}}$ enhancement. It is well established that the exchange bias is the result of an interfacial interaction $^{2,3}$ between a ferromagnet $(\mathrm{F})$ and an antiferromagnet (AF), although the details of the microscopic mechanism are currently widely debated. The situation is complicated as one find that there are a number of parameters ${ }^{4}$ which influenc the exchange bias, such as the anisotropy, crystal structure, ${ }^{5,6}$ interface roughness, interfacial spin configuration and magnetic domains. ${ }^{7}$

Experimental work to date has revealed that $H_{\mathrm{ex}}$ is typically two orders of magnitude smaller than values predicted using the original model proposed by Meiklejohn and Bean, ${ }^{1}$ where it was suggested that the biasing was a consequence of the competition between the Zeeman and the exchange coupling energy across an ideal, smooth, uncompensated interface. Experimentally, however, it is known that biasing does exist at compensated interfaces and in noncrystalline materials. ${ }^{4}$

Two main theoretical approaches have been pursued in an attempt to explain this discrepancy between the predicted and experimental values. Mauri et al. extended the idea of planar domain walls originating at a smooth $\mathrm{AF}$ interface, ${ }^{2}$ where the AF spins rotate in the plane, originally put forward by Néel. ${ }^{8,9}$ The exchange energy in this case is spread across the width of the domain wall. Subsequent models ${ }^{10-12}$ which have extended the Mauri concept have also shown limited success in fully explaining the exchange bias effect. In contrast, Malozemoff ${ }^{13}$ argued that an ideal interface was unrealistic, and roughness leading to magnetic defects gave rise to local random fields The total energy of the AF, including the contribution from the random fields is minimized by the formation of domains in the AF, which have domain walls perpendicular to the interface. Both theories have produced values for $H_{\mathrm{ex}}$ that compare well with experiment under certain conditions, whereas the $H_{\mathrm{c}}$ enhancement has been attributed to the formation of domains in the AF layer. ${ }^{14}$ However, no theory has so far been able to adequately explain $H_{\mathrm{ex}}$, the $H_{\mathrm{c}}$ enhancement, and their temperature and AF thickness dependences in full. 15,16

Due to the nature of the spin structure of an AF, it is not a straightforward process to characterize such layers using conventional magnetization techniques. Neutron diffraction studies on $\mathrm{Fe}_{3} \mathrm{O}_{4} / \mathrm{NiO}$ exchange biased superlattices ${ }^{17}$ have shown that the exchange biasing is related to the formation of both parallel and perpendicular domains, which are frozen into the AF layer on cooling through the blocking temperature $\left(T_{\mathrm{B}}\right)$ - this is the temperature above which $H_{\mathrm{ex}}$ is zero. Similar measurements on $\mathrm{FeMn} / \mathrm{Co}$ superlattices ${ }^{18}$ have shown that a complex random domain structure is found to be present when the magnetization of the Co layer is reversed and is dictated by the underlying random domain structure within the FeMn. The use of nonmagnetic impurities in the AF layer ${ }^{19}$ to form and influenc domains has also highlighted the domain structure in the AF being the cause of biasing, and the mechanism which seems to control it.

Investigations of the AF layer thickness dependence in order to establish the thickness at which the biasing saturates are often made at room temperature, motivated by technological concerns, although some experimental data do exist where the work was carried out at low temperatures. ${ }^{20-23}$ Results so far have been interpreted in terms of parallel domain walls in the $\mathrm{AF} .{ }^{14}$ In this paper we will present evidence that the dependence of $H_{\mathrm{ex}}$ on the AF layer thickness and its variation with temperature in fact supports the idea of perpendicular domain walls. To do this we have used calculations from a more recent microscopic domain model, ${ }^{19,24-26}$ which is able to describe the experimental features we have found at all temperatures through the use of Monte Carlo methods. The model is based on an irreversible $\mathrm{AF}$ interface magnetization which arises from volume domains originating from defects in the bulk of the AF. These volume domains then influenc the spin structure at the interface.

\section{EXPERIMENTAL TECHNIQUES}

The $\mathrm{Co} / \mathrm{Ir}_{25} \mathrm{Mn}_{75}$ system was studied experimentally within a spin-valve structure. This allowed the free Co layer 
within the spin valve to be used as a control layer to which the biased Co layer could be directly compared, while having only a minimal effect on the properties of the pinned layerorange-peel coupling field were $\sim$ a few Oe. It also allowed magnetotransport measurements to be performed, the resistance measurements were done using a standard four point probe de technique. Typical $(300 \mathrm{~K})$ magnetoresistances of our spin valves were $\sim 7 \%$, while typical $(300 \mathrm{~K})$ sheet resistances were $\sim 10 \Omega / \square$. The specimens were deposited by magnetron sputtering at an argon working pressure of 2.5 mTorr. Each set of specimens consisted of 15 samples which were grown during the same vacuum cycle. The base pressure prior to the deposition was of the order of $2 \times 10^{-8}$ Torr. The spin-valve structures $\mathrm{Ta}(75 \AA) / \mathrm{Co}(40 \AA) / \mathrm{Cu}(23 \AA) /$ $\mathrm{Co}(26 \AA) / \operatorname{IrMn}\left(t_{\mathrm{AF}}\right) / \operatorname{Ta}(25 \AA)$ were deposited onto silicon (100) substrates in an in-plane forming fiel of magnitude 200 Oe at ambient temperature. The IrMn layers were deposited from a Mn target with chips of Ir attached to its surface, energy dispersive $\mathrm{x}$-ray absorption spectroscopy yields a composition in the deposited fil of $\sim \mathrm{Ir}_{25} \mathrm{Mn}_{75}$. Deposition rates were determined by measuring the thickness of calibration film by low angle x-ray reflectometr . Measurements of individual layer thicknesses within spin valves by this technique is not straightforward, ${ }^{27}$ but the overall stack heights were consistent with the total nominal thicknesses of the samples. The sample size was $10 \times 2 \mathrm{~mm}^{2}$, smaller than the confine plasma of our two in. magnetron sources, ensuring that there are no thickness gradients across the sample. X-ray diffraction shows that such samples are predominantly (111) textured.

The forming fiel induced a uniaxial anisotropy in the free Co layer and set the pinning direction of the Co layer in contact with the IrMn layer. Magnetic characterization was done using a vibrating sample magnetometer (VSM) (2-300 $\mathrm{K}$ ), and a Magneto-optical kerr effect (MOKE) apparatus equipped with a custom built heater stage for temperatures from $300 \mathrm{~K}$ upwards. No degradation in properties was found after heating, showing that the Ta capping layer used to protect the samples was sufficien to prevent oxidation of the film during the heating process. In the temperature ranges investigated here, changes in the magnetic properties were insignifican after subsequent heating and cooling cycles provided the same fiel cooling procedure was used in each case. For the temperature dependence measurements, all samples were cooled down to $2 \mathrm{~K}$ in a $-0.4 \mathrm{~T}$ field so samples with blocking temperatures below room temperature had their pinning direction set. This procedure means that the Co layer is always completely saturated when the IrMn orders, and tests have shown that a pinned layer set at room temperature shows the same biasing properties when heated above the blocking point and then fiel cooled at $-0.4 \mathrm{~T}$. (Throughout this paper $H_{\mathrm{ex}}$ is a positive quantity, always opposite in sign to the cooling or setting field ) No dependence of $H_{\mathrm{ex}}$ on the magnitude of the cooling fiel was found provided that this fiel was large enough to completely saturate the Co layer. Temperature dependence measurements were then done as a series of hysteresis loops at increasing temperatures. Example loops from both these measurement techniques are shown in Fig. 1. In panels (a) and (d) the
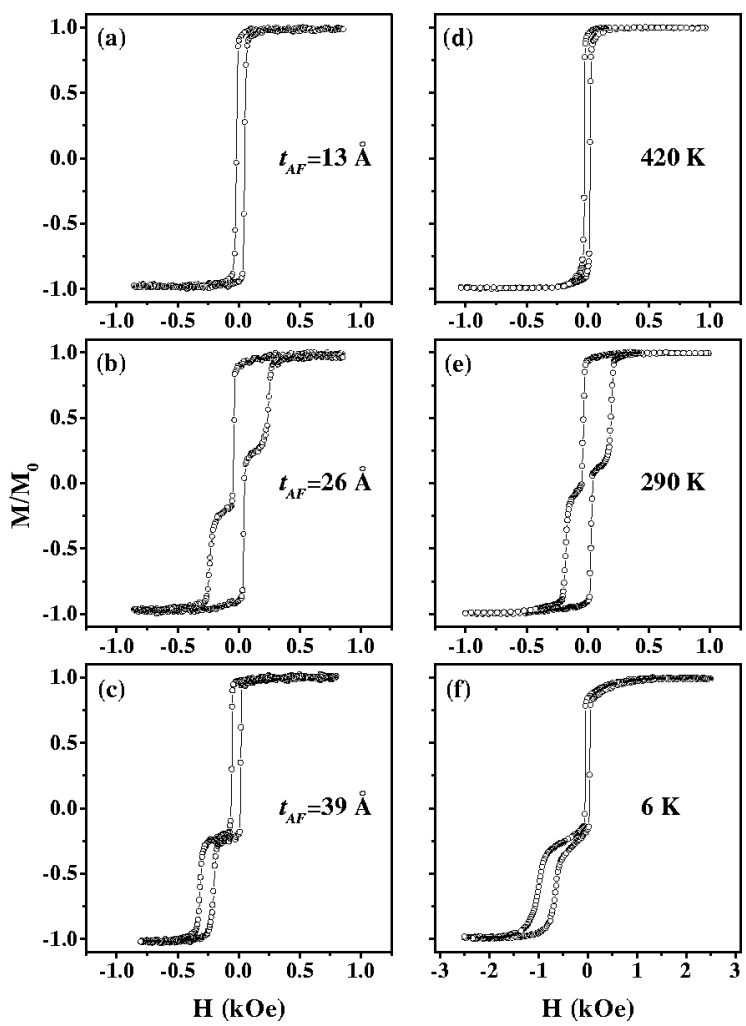

FIG. 1. Representative magnetization loops. Loops (a)-(c) were measured at $295 \mathrm{~K}$ for an IrMn layer thickness $t_{\mathrm{AF}}$ indicated, while loops (d)-(f) were taken at the temperatures indicated for a sample with $t_{\mathrm{AF}}=26 \AA$. Curves (a), (b), (c), and (f) were measured by VSM, while curves (d) and (e) were measured by MOKE.

equivalence of $H_{\mathrm{c}}$ in the free and pinned layers can be seen as the entire sample switches together. Meanwhile in the lower four panels (b), (c), (e), and (f) the switching of the free and pinned layers can be clearly separated, with the free layer identifie by its slightly larger magnetic moment and much softer magnetic properties. This allows us to determine $H_{\text {ex }}$ and $H_{\mathrm{c}}$ for this layer alone by measuring the field at which it switches: as is conventional, $H_{\mathrm{ex}}$ is the average of these two fields while $H_{\mathrm{c}}$ is half the difference between them.

\section{EXPERIMENTAL RESULTS AND DISCUSSION}

In Fig. 2, the temperature dependence of $H_{\mathrm{ex}}$ and $H_{\mathrm{c}}$ of some representative samples are shown for various IrMn layer thicknesses. $H_{\text {ex }}$ of all the samples falls with temperature and vanishes at $T_{\mathrm{B}} . H_{\mathrm{c}}$ of the pinned Co layers shows a peak at $T_{\mathrm{B}}$ and a sharp upturn as very low temperatures are approached. There is no feature in the $H_{\text {ex }}$ plot that accompanies this divergence below $\sim 20 \mathrm{~K}$. The free-layer coercivity increases approximately linearly from its room temperature value of $30 \mathrm{Oe}$ to $50 \mathrm{Oe}$ at $2 \mathrm{~K}$. It is important to note that the pinned layer coercivity at high temperatures matches that of the free layer very closely, indicating comparable intrinsic magnetic properties. With this in mind, it can be seen that the trends observed in the biased Co layers are therefore not due to the magnetization or anisotropy constant of $\mathrm{Co}$ 

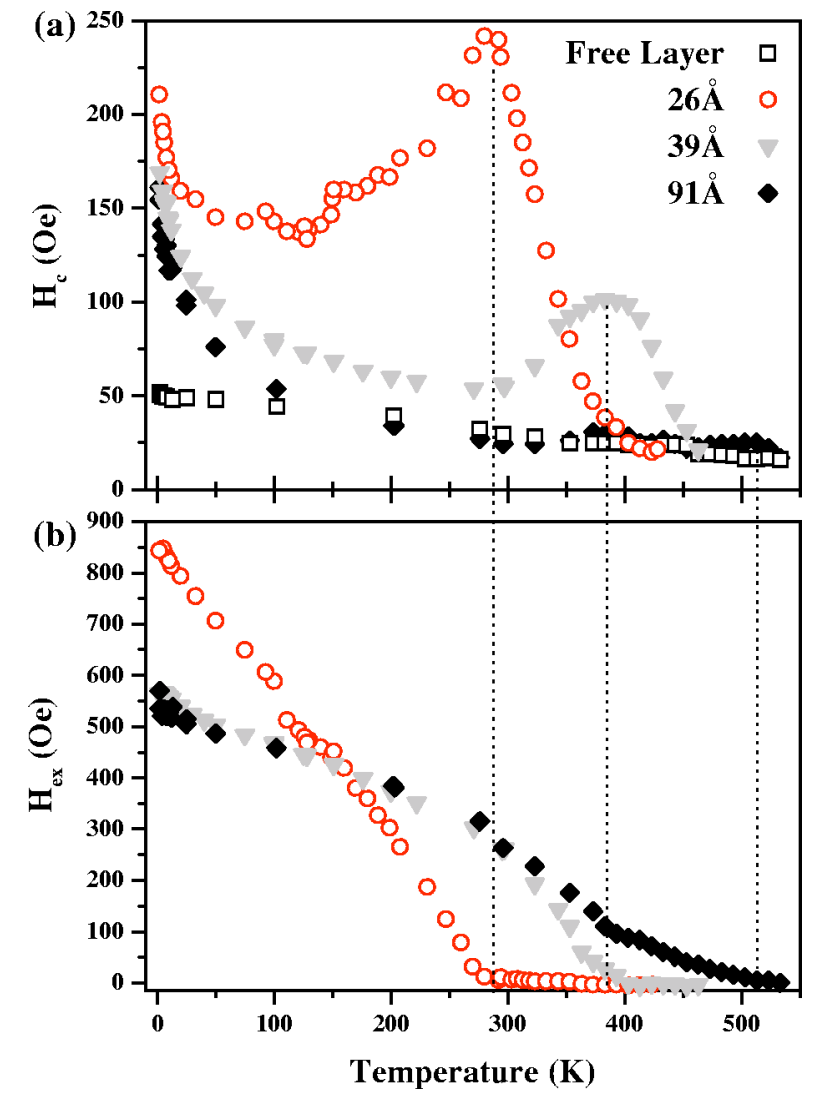

FIG. 2. Temperature dependence of the coercivity $H_{\mathrm{c}}$ (a) and exchange bias fiel $H_{\mathrm{ex}}$ (b) for selected IrMn thicknesses. The open square symbols represent the coercivity of the free Co layer within the spin valve. Samples were initially fiel cooled in $0.4 \mathrm{~T}$ fiel to $2 \mathrm{~K}$ before commencing measurements. The vertical dashed lines indicate the blocking temperatures of the three samples.

itself varying significantl with temperature. Hence the changes must be occurring as a result of what is occurring in the AF layer.

Naively, one might expect $H_{\mathrm{ex}}$ to fall off with temperature in proportion to the AF order parameter of IrMn, going to zero at the Néel temperature $T_{\mathrm{N}}$. From Fig. 2(b) this is not obviously the case, $H_{\mathrm{ex}}$ is found to decrease approximately linearly to zero at $T_{\mathrm{B}}$ for the sample containing the 14- $\AA$-thick IrMn layer.

Similar peaks in $H_{\mathrm{c}}$ at $T_{\mathrm{B}}$ observed in oxidized NiFe layers have been explained using a thermal fluctuatio model by Fulcomer and Charap. ${ }^{15}$ They assumed that the small oxide particles only coupled to the magnetic NiFe fil and were independent of each other. In this form the varying sizes of particles would lead to superparamagnetism. In the system studied here, it would be very unlikely that the grains would behave independently of each other because of the dense, continuous nature of the film A more recent mean-fiel theory by Wee et al., ${ }^{16}$ which considers an epitaxial system, has shown similar results for uncompensated interfaces based on a parallel domain wall. They predict a blocking temperature that arises due to thermal dissipation of a domain wall, where $T_{\mathrm{B}}$ is the temperature at which the domain wall is no longer sustainable. Stiles and McMichael give a description of peaks in $H_{\mathrm{c}}$ which combines aspects of both the theories described above using a mesoscopic model assuming effective magnetic moments for the grains of the $\mathrm{AF}^{28}$ In general what is important is that in this range of temperatures, a large fraction of the hysteretic losses occur in the AF layer, giving rise to an enhanced coercivity.

That an interaction between the two layers still exists above $T_{\mathrm{B}}$ can be seen from the significantl enhanced $H_{\mathrm{c}}$ as shown in Fig. 2(a). The true nature of this interaction is open for debate. In models such as those mentioned above, as well as our own, above $T_{\mathrm{B}}$ the entire spin structure of the AF layer reverses with the ferromagnet as the energy barrier holding it in place can be overcome by the torque exerted by the $\mathrm{F}$ spins once it has dropped below a certain height. One might believe that the temperature at which $H_{\mathrm{c}}$ drops to the intrinsic value seen in the free layer sets a lower limit on $T_{\mathrm{N}} \cdot{ }^{29}$ However, one cannot exclude that an AF even in its paramagnetic phase has some influenc on the reversal of the F. The AF experiences an interfacial exchange fiel originating from the $\mathrm{F}$ and a paramagnet in a fiel has a magnetization. Of course, it must follow the F during reversal, which means that it is reversible, and will yield zero $H_{\mathrm{ex}}$ but possibly an enhanced $H_{\mathrm{c}}$.

Experimentally, there are two temperatures which are important. These are $T_{\mathrm{B}}$, which is the point at which $H_{\mathrm{ex}}$ tends to zero, and the temperature at which $H_{\mathrm{c}}$ of the biased layer falls to that of the free layer which we assume to be just below or equal to $T_{N}$, subject to the caveats given above. The size and width of the coercive peak is found to decrease, as does the difference between $T_{\mathrm{B}}$ and $T_{\mathrm{N}}$, as the IrMn layer is made thicker. This effect is similar to that reported recently in the $\mathrm{MnF}_{2} / \mathrm{Fe}$ system by Leighton et al., ${ }^{30}$ where the peak in $H_{\mathrm{c}}$ was observed to be suppressed for very high AF layer thicknesses. This was explained within the Stiles-McMichael model ${ }^{28}$ as being determined by within which layer (AF or F) the hysteretic losses predominantly occur. This would seem to imply that some energy term which is volume dependent is becoming more important in determining $T_{\mathrm{B}}$. We fin that $T_{\mathrm{B}}$ and $T_{N}$ converge at $550 \mathrm{~K}, T_{N}$ of bulk IrMn, for very thick IrMn layers. Until recently, it was thought that the reduction in $T_{\mathrm{B}}$ due to a lowering of the AF layer thickness was a consequence of a reduction in the ordering temperature of AF $\left(T_{N}\right)$. This was assumed to be a result of finite-siz scaling effects. ${ }^{22}$ However, it has been shown in neutron diffraction studies that $T_{\mathrm{B}}$ does not follow $T_{N}$, and moreover superparamagnetic effects due to grains cannot fully explain the behavior. ${ }^{31}$

The IrMn thickness and temperature dependence of both the exchange bias fiel and coercivity are shown in Fig. 3. At room temperature the onset of exchange biasing appears at a critical AF layer thickness of $21 \AA$ and continues to increase up to a layer thickness of $40 \AA$, where it saturates at a value of 270 Oe. These finding are similar to results obtained previously that were explained by the idea of a partial domain wall ${ }^{14}$ in the AF parallel to the interface. On this basis one would expect a wall thickness in IrMn of only some $40 \AA$. In contrast, as the temperature is decreased down to 2 $\mathrm{K}$, which removes almost all thermally activated processes, 


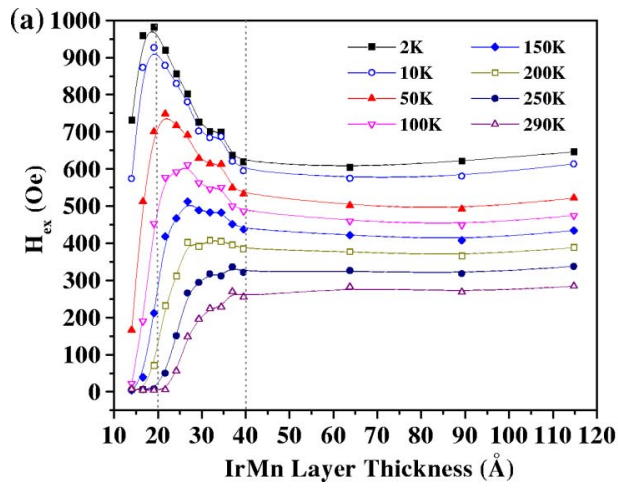

one find that the critical thickness for the onset of biasing is less than $14 \AA$, our thinnest sample. At about this thickness $H_{\text {ex }}$ rapidly increases to 950 Oe where it peaks at $20 \AA$, before falling to a constant value of $\sim 620$ Oe above $40 \AA$. At this stage one might consider the possibility that the variations in the value of $H_{\mathrm{ex}}$, with their steep temperature dependence, were related to the discontinuity of layers thinner than $t_{\mathrm{AF}} \approx 40 \AA$. Under the same growth conditions used to prepare the IrMn layers in our spin valves, we deposited IrMn on a piece of thermally oxidized Si while making an in situ four-point resistance measurement. This fil was subsequently subjected to x-ray reflectometr to determine its total thickness and hence the rate of deposition. We found that the fil becomes conducting (viz. the percolation transition takes place) at an equivalent thickness of $\sim 11 \AA$. While complete continuity will require a few Angstrom more, the superior wetting of metal-on-metal growth in our spin-valve structures will mean that a few Angstrom less are needed. These corrections will roughly cancel, leaving us with a $t_{\mathrm{AF}}$ for continuity well below the thickness where variations in $H_{\text {ex }}$ set in at any temperature.

The exchange fiel at low temperatures exhibits very similar features to the predictions of the random-fiel model proposed by Malozemoff. ${ }^{13}$ The low thickness onset of $\sim 10 \AA$ for exchange biasing questions the existence of parallel domains - can the wall be so thin? — while the overall shape of $H_{\mathrm{ex}}\left(t_{\mathrm{AF}}\right)$ favors the idea of an in-plane domain structure due to interface roughness ${ }^{13}$ or due to volume defects. ${ }^{24}$ A similar peaked form to the $H_{\mathrm{ex}}\left(t_{\mathrm{AF}}\right)$ curve was observed at room temperature in a very similar materials system ( $\mathrm{IrMn} / \mathrm{CoFe}),{ }^{32}$ but in that instance the peak was ascribed to the variation of (111) texture with IrMn thickness. These samples differ from our own in that IrMn was deposited prior to CoFe that is to be pinned, while it was also buffered with a magnetic material, permalloy, so some differences in microstructure are to be expected. Since we are able to reversibly suppress the peak in our data simply by varying the temperature, it seems that its presence is related to variations in the magnetic, rather than the physical microstructure of our spin valves. The complex behavior within this set of data cannot be fully explained using existing models.

Turning our attention to $H_{\mathrm{c}}$ of these layers, it is interesting to note that $H_{\mathrm{c}}$ begins to increase with IrMn layer thickness before any sign of biasing at all temperatures, as seen in Fig. $3(\mathrm{~b})$. As this occurs even at $2 \mathrm{~K}$, we believe that this is
FIG. 3. IrMn thickness dependence of the exchange bias fiel $H_{\text {ex }}$ (a) and coercivity $H_{\mathrm{c}}$ (b) for a number of temperatures. Lines between the points are a guide to the eye. inconsistent with the idea of the $H_{\mathrm{c}}$ peak arising from superparamagnetic effects, as the thermal fluctuation will be too small. The AF interfacial and bulk spin structures are reversible until the AF anisotropy energy barrier is sufficientl large to stabilize them against torques from the rotating $\mathrm{F}$ spins to which they are coupled. This leads to additional energy dissipation in the AF layer, manifesting itself as additional coercivity. The peak in coercivity correspondingly shifts to smaller thicknesses consistent with the exchange bias onset. It is clearly demonstrated that the peaks found in the enhanced $H_{\mathrm{c}}$ are an intrinsic part of the exchange bias. Comparing this discussion of the peak in $H_{\mathrm{ex}}$ with $t_{\mathrm{AF}}$ with that given above for the peak in $H_{\mathrm{ex}}$ with $T$ it is clear that it is possible to draw parallels between the manner in which biasing appears as function of $t_{\mathrm{AF}}$ and the way it vanishes at $T_{\mathrm{B}}$ - there is a conceptual similarity between rising temperature and falling AF layer thickness.

\section{MODELING}

Naturally it is desirable to make the qualitative arguments above more rigorous. The most striking feature of the experimental data is the appearance of the peak in $H_{\mathrm{ex}}$ at low $t_{A F}$ and its suppression on warming the sample. In the past, only the model of Malozemoff ${ }^{13}$ predicted such a peak, but since it is a zero-temperature model it cannot account for the changes as the temperature is raised. We have therefore made use of the so-called domain state model, ${ }^{19,25,26}$ which also takes into account disorder in the sample, but makes use of Monte Carlo techniques to treat finit temperature. The model consists of one monolayer of a $\mathrm{F}$ and $t$ monolayers of AF (see Ref. 26 for a sketch of the model). To include a certain amount of structural disorder the AF is diluted or, in other words, a fraction $p$ of randomly chosen sites of the AF are left without a spin. The $\mathrm{F}$ is exchange coupled to the topmost layer of the AF. Previously the model has been used to simulate experimental systems where the AF is deliberately diluted by growth using $\mathrm{Mg}$ impurities or oxygen defi ciency in CoO. ${ }^{19}$ However, at the heart of the model is the idea that a number of exchange bonds in the AF are broken at random, leading to places where domain walls can form at a reduced energy cost. In the experimental system in question at present, which is a random substitutional alloy, one would expect that Ir-Mn or Ir-Ir exchange bonds will be substantially weaker than a Mn-Mn bond. We therefore ex- 
pect that the model is a useful approximation to reality in this particular case.

The Hamiltonian $\mathcal{H}$ of the system is written as

$$
\begin{aligned}
\mathcal{H}= & -J_{\mathrm{F}} \sum_{\langle i, j\rangle} \vec{S}_{i} \cdot \vec{S}_{j}-\sum_{i}\left(d_{z} S_{i z}^{2}+d_{x} S_{i x}^{2}+\vec{S}_{i} \cdot \vec{B}\right) \\
& -J_{\mathrm{AF}} \sum_{\langle i, j\rangle} \epsilon_{i} \epsilon_{j} \vec{\sigma}_{i} \cdot \vec{\sigma}_{j}-\sum_{i} \epsilon_{i}\left(k_{z} \sigma_{i z}^{2}+\vec{\sigma}_{i} \cdot \vec{B}\right) \\
& -J_{\mathrm{INT}} \sum_{\langle i, j\rangle} \epsilon_{j} \vec{S}_{i} \cdot \vec{\sigma}_{j},
\end{aligned}
$$

where $\vec{S}_{i}$ and $\vec{\sigma}_{i}$ denote spins at the $i$ th site corresponding to $\mathrm{F}$ and AF, respectively. The magnetic fiel $\vec{B}$ is applied along the $z$ direction, while the $x$ direction is normal to the layers. The firs line of the Hamiltonian describes the energy of the $\mathrm{F}$ with the $z$ axis as its easy axis with an anisotropy constant $d_{z}>0$ and the $x$ axis as its hard axis with an anisotropy constant $d_{x}<0$. The resulting in-plane anisotropy keeps the $\mathrm{F}$ preferentially in the $y-z$ plane. The second line is the contribution from the $\mathrm{AF}$ with quenched disorder $\left(\epsilon_{i}=0,1\right)$ also having its easy axis along $z\left(k_{z}=J_{\mathrm{F}}>0\right)$. The last term describes the interaction of the $\mathrm{F}$ with the interface $\mathrm{AF}$ monolayer.

We consider nearest-neighbor interactions on a simple cubic lattice with exchange constants $J_{\mathrm{F}}$ and $J_{\mathrm{AF}}$ for the $\mathrm{F}$ and the AF, respectively, while $J_{\text {INT }}$ stands for the exchange constant between $\mathrm{F}$ and AF. In our simulations we set $J_{\mathrm{F}}$ $=-2 J_{\mathrm{AF}}=2 J_{\mathrm{INT}}$, broadly comparable with the ratios of these values that would be expected in the experimental system. The anisotropy constants are scaled up compared to the experimental system in order to observe the relevant phenomena in much thinner layers in order to keep the computational demands within realistic limits.

Starting from a temperature $T$ above the Néel temperature $T_{N}(p)$ of the diluted AF but below the Curie temperature $T_{c}$ of the $\mathrm{F}$, the system is cooled below $T_{N}$ in presence of an external magnetic fiel $\vec{B}=0.25 J_{\mathrm{F}} \hat{z}$. The fina temperature of the whole system is varied from $T=0.05 J_{\mathrm{F}}$ to $T=0.5 J_{\mathrm{F}}$, roughly the temperature range that was experimentally studied according to our energy scale given above. At this temperature the hysteresis curve of the system is calculated along the $\hat{z}$ direction. The results presented in this paper were obtained with $p=0.4$. This is a value for the dilution which is well below the percolation limit so that the AF does not split into isolated clusters or grains but consists mainly of an interacting, structurally disordered antiferromagnetic crystal.

Figure 4(a) shows the behavior of the bias fiel as a function of the AF thickness $t$ at different temperatures. The model correctly reproduces the appearance of a peak in the exchange bias at some intermediate thickness as was also experimentally observed [see Fig. 3(a)]. Our calculations also show that the peak shifts towards higher values of the $\mathrm{AF}$ thickness with increasing temperature and simultaneously the strength of the peak is reduced, just as in the experiment.
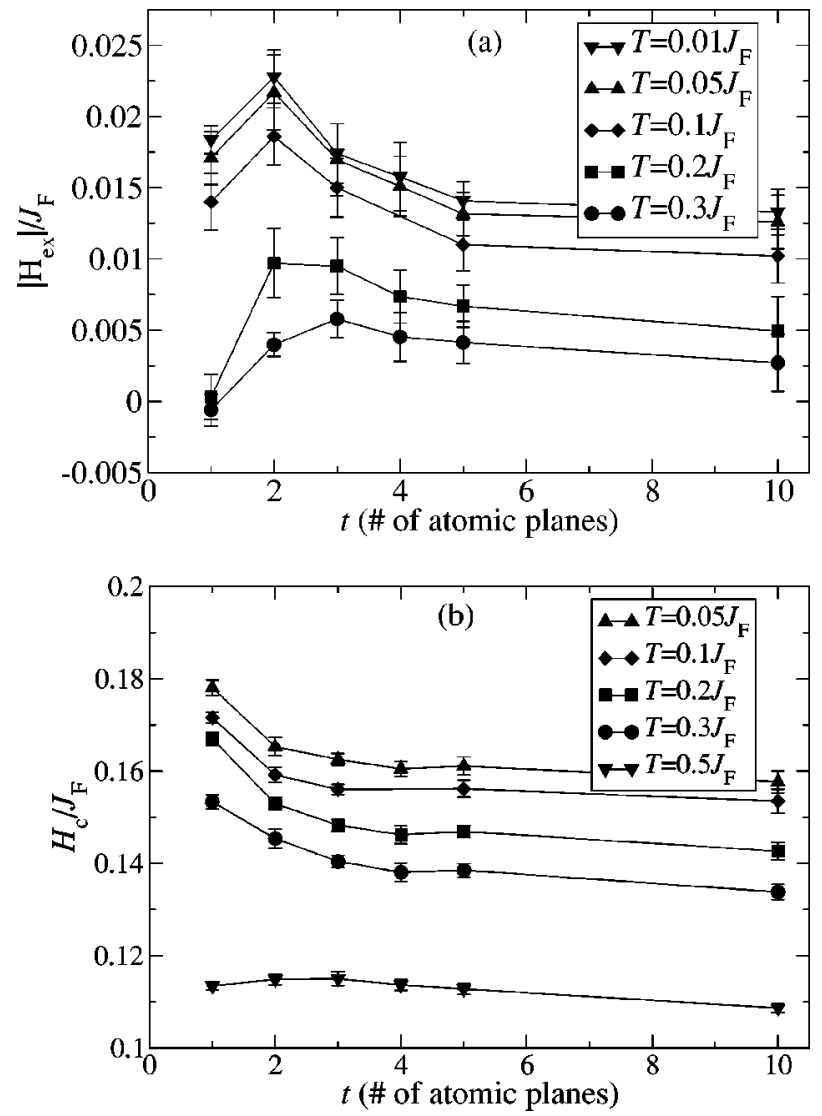

FIG. 4. Prediction of the AF thickness dependence of (a) the exchange bias fiel and (b) the corresponding prediction of the coercivity from the stability analysis of the interface AF domains at different temperatures.

These finding can be understood from the behavior of the AF. As was shown earlier, ${ }^{19,24,26}$ during a fiel cycle, the interface magnetization of the AF displays a hysteresis following the $\mathrm{F}$ due to the interface coupling. Additionally, the whole curve is shifted vertically due to the fact that after fiel cooling the AF is in a domain state with a partly frozen surplus magnetization. This shift of the entire hysteresis loop of the AF proves the existence of an irreversible magnetization stored in the AF domains. While the irreversible domain state magnetization of the AF acts as an additional effective fiel on the F, resulting in exchange bias, the reversible part of the AF magnetization is responsible for the enhanced coercive fiel of the F.

The rapid increase of exchange bias starting from very thin film can be explained by the fact that there is a critical thickness for domain wall stability. Below this thickness the domain state magnetization in the $\mathrm{AF}$ is reversible, giving rise to additional coercivity without bias. At the critical thickness, some of the domain state magnetization rapidly becomes irreversible leading to bias and a decrease in coercivity. Above this thickness the decline in $H_{\text {ex }}$ is caused by the fact that with increasing AF thickness it becomes more and more difficul to form domain walls since these are oriented perpendicular to the interface extending through the whole AF layer. ${ }^{26}$ The corresponding domain-wall energy in- 
creases with thickness but can be reduced by reducing the number of domain walls, i.e. by the formation of larger domains (see Fig. 2 in Ref. 24) which then reduce the interface magnetization and, hence, the bias field

These two competing effects-the initial amount of interface magnetization versus its stability - give rise to the peak of $H_{\mathrm{ex}}$ at some intermediate value of thickness. Also, the temperature $T$ of the system plays a crucial role for the stability of the AF interface magnetization. As we increase $T$, the interface becomes more and more unstable because of enhanced thermal fluctuations Consequently, more monolayers of $\mathrm{AF}$ are required to stabilize the interface, and hence the peak is shifted towards higher values of $t_{\mathrm{AF}}$ at higher temperatures. However, beyond a certain temperature the interface becomes too unstable and therefore the exchange bias disappears. Thus the blocking temperature, define as the temperature at which exchange bias disappears, can be interpreted as the temperature above which the interface monolayer of the AF loses its stability during hysteresis. There are examples in the literature of materials systems where the peak is not observed at any temperature, and we are able to adjust our model to account for this simply by varying the value of $p$.

The behavior of the coercive fiel is qualitatively different [see Fig. 4(b)]. It follows the reversible part of the interface magnetization of the AF because it is influence by the uniaxial (rather than unidirectional) anisotropy of the AF which, via its interface magnetization, is transferred to the F. This effect depends on the amount of interface magnetization only, and not on its stability which - as explained above-is important for the exchange bias field Consequently, the coercive fiel is greater for thinner layers even for lower thicknesses where the exchange bias fiel is shrinking. For higher temperatures, however, the AF might already be paramagnetic for the lowest thickness we simulated leading neither to exchange bias nor to an enhanced coercivity. When com- pared to the experimental data of Fig. 3(b), we can see that again the theory reproduces the experimentally observed phenomena: the coercivity falls with rising temperature, and a peak is observed in $H_{\mathrm{c}}$ at the onset thickness of exchange bias. Only the very sharp upturn in $H_{\mathrm{c}}$ at low temperatures seen in the experimental data is not fully reproduced within this model, and here further refinement are necessary.

\section{CONCLUSION}

To conclude, we have shown that the AF thickness dependence of the exchange bias, when studied at various temperatures, yields a very rich phenomenology. At low temperatures $H_{\text {ex }}$ sets in at very low thicknesses and then passes through a sharp peak before decaying to a constant value that is maintained out to the largest thicknesses we have measured. As the temperature is raised, the onset thicknesses rises, the peak moves to higher thicknesses and is gradually suppressed, and the high thickness value falls. Any theory that hopes to describe exchange bias should be able to reproduce all the observed features. In addition to the difficultie associated with fittin a planar domain wall into an AF layer only 14- $\AA$ thick, such models are unable to succeed in predicting a peak in $H_{\mathrm{ex}}$. Comparison of the experiments and calculations presented in this paper strongly favors the idea of an in-plane domain structure being an essential ingredient for a realistic description of biasing. Coercivity enhancement at the onset or disappearance of $H_{\mathrm{ex}}$ (due to either thickness or temperature) is intrinsic to biasing and is due to reversible pinning. No other model available at the present time is able to reproduce all of these effects together.

\section{ACKNOWLEDGMENTS}

This work was supported by the EPSRC and Seagate Technology at Leeds, and by the Deutsche Forschungsgemeinschaft through SFB 491 at Duisburg.
*Email address: phyma@phys-irc.leeds.ac.uk; URL: http:// www.stoner.leeds.ac.uk

${ }^{\dagger}$ Present address: Department of Physics and Astronomy and MINT Center, University of Alabama, Tuscaloosa, AL 35487.

${ }^{1}$ W.H. Meiklejohn and C.P. Bean, Phys. Rev. 105, 904 (1957).

${ }^{2}$ D. Mauri, H.C. Siegmann, P.S. Bagus, and E. Kay, J. Appl. Phys. 62, 3047 (1987).

${ }^{3}$ C. Tang, N. Heiman, and K. Lee, J. Appl. Phys. 52, 2471 (1981).

${ }^{4}$ J. Nogues and I.K. Schuller, J. Magn. Magn. Mater. 192, 203 (1999); A.E. Berkowitz and K. Takano, ibid. 200, 552 (1999).

${ }^{5}$ K. Takano, R.H. Kodama, A.E. Berkowitz, W. Cao, and G. Thomas, Phys. Rev. Lett. 79, 1130 (1997); J. Appl. Phys. 83, 6888 (1998).

${ }^{6}$ M.D. Stiles and R.D. McMichael, Phys. Rev. B 59, 3722 (1999).

${ }^{7}$ A. Scholl, F. Nolting, J. Stöhr, T. Regan, J. Lüning, J.W. Seo, J.-P. Locquet, J. Fompeyrine, S. Anders, H. Ohldag, and H.A. Padmore, J. Appl. Phys. 89, 7266 (2001); W. Kuch, F. Offi L.I. Chelaru, M. Kotsugi, K. Fukumoto, and J. Kirschner, Phys. Rev. B 65, 140408(R) (2002).

${ }^{8}$ L. Néel, Ann. Phys. (Paris) 2, 61 (1967).

${ }^{9}$ Selected Works of Louis Néel, edited by N. Kurti (Gordon and Breach, New York, 1988).
${ }^{10}$ N.C. Koon, Phys. Rev. Lett. 78, 4865 (1998).

${ }^{11}$ T.C. Schulthess and W.H. Butler, Phys. Rev. B 85, 5510 (1999).

${ }^{12}$ T.C. Schulthess and W.H. Butler, J. Appl. Phys. 59, 3722 (1999).

${ }^{13}$ A.P. Malozemoff, Phys. Rev. B 35, 3679 (1987); 37, 7673 (1998); J. Appl. Phys. 63, 3874 (1988).

${ }^{14}$ H. Xi and R.M. White, Phys. Rev. B 61, 80 (2000).

${ }^{15}$ E. Fulcomer and S.H. Charap, J. Appl. Phys. 43, 4184 (1972); 43, 4190 (1972).

${ }^{16}$ L. Wee, R.L. Stamps, and R.E. Camley, J. Appl. Phys. 89, 6913 (2001).

${ }^{17}$ J.A. Borchers, Y. Ijiri, D.M. Lind, P.G. Ivanov, R.W. Erwin, Aron Qasba, S.H. Lee, K.V. O’Donovan, and D.C. Dender, Appl. Phys. Lett. 77, 4187 (2000).

${ }^{18}$ C.H. Marrows, S. Langridge, M. Ali, A.T. Hindmarch, D.T. Dekadjevi, S. Foster, and B.J. Hickey, Phys. Rev. B 66, 024437 (2002).

${ }^{19}$ P. Miltényi, M. Gierlings, J. Keller, B. Beschoten, G. Güntherodt, U. Nowak, and K.D. Usadel, Phys. Rev. Lett. 84, 4224 (2000).

${ }^{20}$ H. Sang, Y.W. Du, and C.-L. Chien, J. Appl. Phys. 85, 4931 (1999).

${ }^{21}$ M.S. Lund, W.A.A. Macedo, K. Liu, J. Nogués, I.K. Schuller, and 
C. Leighton, Phys. Rev. B 66, 054422 (2002).

${ }^{22}$ T. Ambrose and C.-L. Chien, J. Appl. Phys. 83, 6822 (1998).

${ }^{23}$ S.M. Zhou, Kai Liu, and C.L. Chien, J. Appl. Phys. 87, 6659 (2000).

${ }^{24}$ U. Nowak, A. Misra, and K.D. Usadel, J. Appl. Phys. 89, 3874 (2001)

${ }^{25}$ U. Nowak, K.D. Usadel, J. Keller, P. Miltényi, B. Beschoten, and G. Güntherodt, Phys. Rev. B 66, 014430 (2002).

${ }^{26}$ U. Nowak, A. Misra, and K.D. Usadel, J. Magn. Magn. Mater. 240, 243 (2002).

${ }^{27}$ T.P.A. Hase, B.K. Tanner, P. Ryan, C.H. Marrows, and B.J. Hickey, IEEE Trans. Magn. 34, 831 (1998).

${ }^{28}$ M. Stiles and R.D. McMicheal, Phys. Rev. B 63, 064405 (2001).

${ }^{29}$ The exact definitio of the Néel point in exchange bias systems is fraught with greater ambiguity as further rigor is sought. The bulk $T_{\mathrm{N}}$ can be modifie by the finit size of the ultrathin AF layer, as discussed in Ref. 22, making it lower than the bulk value. It may also be modifie by the presence of an exchange fiel from the adjacent ferromagnet, making it higher than the bulk value. An example of this can be seen in Ref. 31. We have made no attempt to treat these proximity effects in this paper.

${ }^{30}$ C. Leighton, M.R. Fitzsimmons, A. Hoffmann, J. Dura, C.F. Majrkzak, M.S. Lund, and I.K. Schuller, Phys. Rev. B 65, 064403 (2002).

${ }^{31}$ P.J. van der Zaag, Y. Ijiri, J.A. Borchers, L.F. Feiner, R.M. Wolf, J.M. Gaines, R.W. Erwin, and M.A. Verheijen, Phys. Rev. Lett. 84, 6102 (2000).

${ }^{32}$ J. van Driel, F.R. de Boer, K.-M.H. Lenssen, and R. Coehorn, J. Appl. Phys. 88, 975 (2000). 